\title{
Sistemática de Commelinales com ênfase em linhagens Neotropicais
}

Marco Octávio de Oliveira Pellegrini

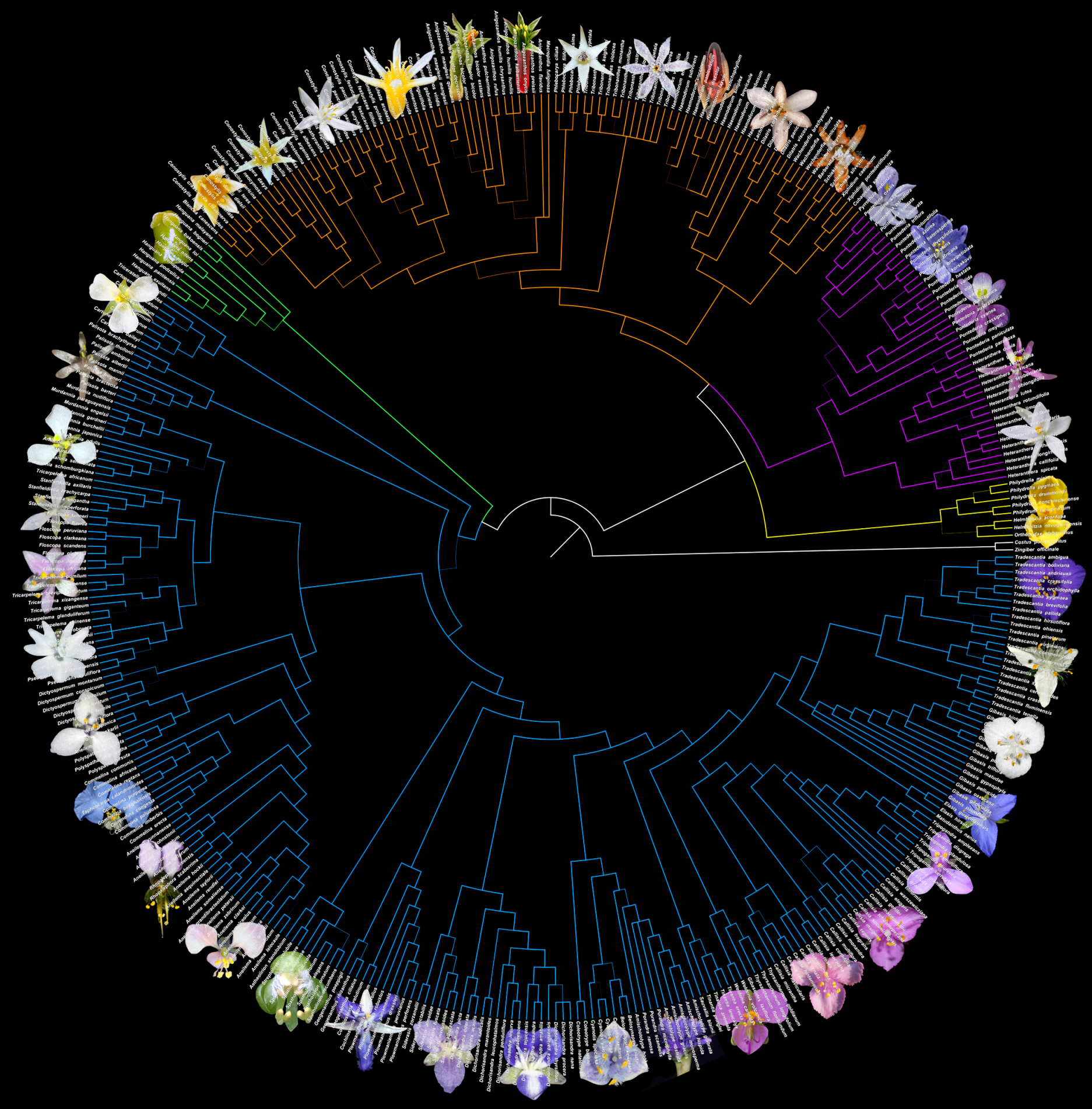

São Paulo 
Marco Octávio de Oliveira Pellegrini

Sistemática de Commelinales com ênfase em linhagens neotropicais

Systematics of Commelinales focusing on neotropical lineages

São Paulo

2019 


\section{Marco Octávio de Oliveira Pellegrini}

\section{Sistemática de Commelinales com ênfase em linhagens neotropicais}

\section{Systematics of Commelinales focusing on neotropical lineages}

Tese apresentada ao Instituto de Biociências da Universidade de São Paulo, para a obtenção de Título de Doutor em Ciências Biológicas, na Área de Botânica.

Orientador: Dr. Jefferson Prado

São Paulo 
Pellegrini, Marco Octávio de Oliveira Sistemática de Commelinales com ênfase em linhagens neotropicais / Marco Octávio de Oliveira Pellegrini; orientador Jefferson Prado. -- São Paulo, 2019.

627 f. + anexo

Tese (Doutorado) - Instituto de Biociências da Universidade de São Paulo, Departamento de Botânica.

1. Commelinales. 2. Taxonomia. 3. Filogenia. 4. Biogeografia. 5. Sistemática. I. Prado, Jefferson, orient. II. Título. 


\section{Comissão Julgadora:}

Prof.(a) Dr.(a)

Prof.(a) Dr.(a)
Prof.(a) Dr.(a)

Prof.(a) Dr.(a)

Dr. Orientador Jefferson Prado 
Aos amores e inspirações da minha vida, meu avô Jorge Felice Pellegrini (in memoriam), e minha afilhada, Roberta de Oliveira Giardini Preisner. Vocês são as luzes que me guiam, sempre fazendo eu me esforçar para ser a melhor versão de mim mesmo. 


\section{EPÍGRAFE}

I don't need you to respect me, I respect me

I don't need you to love, I love me

But I want you to know you could know me

If you change your mind

If you change your mind...

Rebecca Sugar, Steven Universe 


\section{AGRADECIMENTOS}

Essa tese é um reflexo dos vários anos da minha formação acadêmica, sendo o resultado de muita curiosidade e amor pelo meu trabalho. Mais do que isso, essa tese é uma prova de superação pessoal e profissional, mostrando que por maior que sejam os obstáculos colocados na nossa frente, sempre é possível vencê-los. Assim, eu primeiramente gostaria de agradecer aos incontáveis obstáculos dos últimos quatro anos, por terem ajudado a moldar quem eu sou. Graças a vocês, sei quem eu sou, quem eu quero ser, mas principalmente, quem eu nunca quero me tornar! Pois, como disse Christina Aguilera: "Vocês me fizeram ainda mais forte, me fizeram trabalhar mais duro, me deixaram mais sábio, me fizeram aprender mais rápido, tornaram minha pele mais grossa, me ajudaram a me tornar ainda mais inteligente. Então, obrigado por me tornarem um lutador!". Entretanto, apesar de toda a minha superação, peço que "Permitam que eu fale, não as minhas cicatrizes. Elas são coadjuvantes, não, melhor figurantes que nem deviam estar aqui." (AmarELO - Emicida, Majur \& Pabllo Vittar).

Meu mais sincero obrigado ao meu orientador, Dr. Jefferson Prado, por ter me acolhido em um momento de indescritível necessidade. Você me ajudou a alcançar meu sonho de ser doutor em Botânica, esteve do meu lado todas as vezes que precisei, e acreditou e lutou por mim quando vários não o fizeram. Você é uma pessoa que admiro de maneira pessoal e profissional, e espero poder continuar trabalhando contigo! Um obrigado especial por ter tido tanta paciência e dedicação revisando e me ajudando a melhorar todos os meus capítulos da tese, mesmo eles não sendo com samambaias e várias vezes sendo excessivamente longos e complicados.

Agradeço à CAPES pela minha bolsa de doutorado, que me permitiu financiar toda a minha pesquisa e minhas viagens à Europa para visitar herbários. Agradeço também à Fundação Flora de Apoio à Botânica e Smithsonian Institution pela bolsa e suporte financeiro para integrar o programa REFLORA. Essa oportunidade foi decisiva para o sucesso da minha tese. Sou muito grato à todas as instituições que tão solicitamente me receberam e permitiram a realização desse trabalho. Obrigado ao Instituto de Pesquisas Jardim Botânico do Rio de Janeiro, que foi (e sempre será) minha segunda casa, em especial aos funcionários do herbário que sempre estiveram ao meu lado: Dona Luzia, Rosângela, Luís Fernando, Clarisse e Érika. Agradeço também ao Instituto de Botânica, Jardim Botânico de São Paulo), por me ceder seu espaço e infraestrutura para a 
conclusão dessa tese, especialmente à Candinha Mamede por auxílio com os empréstimos e Margarida Fiuza por sempre me receber tão bem. Agradeço de coração à Denise Trombert e Elder Paiva, da Universidade Federal de Minas Gerais por me permitirem utilizar as instalações do Laboratório de Secreção e Reprodução de Vegetais, sempre que eu precisei. Também sou imensamente grato ao Smithsonian Institution, que me recebeu durante um ano como pesquisador visitante e fez com que eu me sentisse sempre em casa e bem-vindo. Agradecimentos especiais para Laurence Dorr (former department chair), Eric Schuettpelz (department chair), Mary Ann Apicelli (administrative specialist), Leslie Brothers (greenhouse manager), Rusty Russell e Sue Lutz (collection managers), Mary Sangrey (academic services), Carol Kelloff, Erika Gardner, Meghann Toner, Melinda Peters e Nancy Khan (collection support), Chris Tuccinardi, Ingrid P. Lin e Sylvia Orli (information management), Ida Lopes, Mark Strong e Stanley Yankowski (research assistants), Gary Krupnick (plant conservation unit), Robin Everly (botany library), Alice Tangerini (illustrator), John Kress, Jun Wen, Kenneth Wurdack, Pedro Acevedo, Warren Wagner e Vicki Funk (curators), John Wiersema e Joseph Kirkbride (research associate), e Harold Robinson e Robert Faden (research emeritus). Também agradeço à Grand Valley State University por me receber e alojar para o desenvolvimento da parte molecular da minha tese. Finalmente, agradeço aos funcionários do Royal Botanic Gardens, Kew e Muséum National d'Histoire Naturelle por me receberem tão bem durante minhas visitas. Também sou muito grato a todos os meus coautores e colaboradores, que foram parte essencial nos resultados dessa tese: Adolfo Espejo Serna (Universidad Autónoma Metropolitana-Iztapalapa), Charles Horn (Newberry College), Ehoarn Bidault (Muséum National d'Histoire Naturelle), JeF Veldkamp (in memoriam), Jorge Guttiérrez (Jardín Botánico Nacional, Cuba), Kate Hertweck (Fred Hutchinson Cancer Research Center), Maria Luiza de Carvalho (UFBA), Matti Niissalo (Singapore Botanic Gardens), Mayur Nandikar (Naoroji Godrej Centre for Plant Research), Rajaram Gurav (Shivaji University), Rhian Smith (Royal Botanic Gardens, Kew), Robert Faden e Kenneth Wurdack (Smithsonian Institution), Stephen Hopper e Ellen Hickman (University of Western Australia), e Timothy Evans (Grand Valley State University).

Sou imensamente grato aos meus amigos que me acompanharam nessa jornada! Sei que a pós-graduação me fez excessivamente ausente e furão, mas agradeço a 
compreensão, o amor e todo o apoio que vocês sempre me dão. Saibam que os agradecimentos aqui são enxutos por limitações físicas, mas espero sempre ter deixado muito claro o quanto eu amo todos vocês! Muito obrigado aos meus amigos de longa data: Filipe Duarte, Ísis Daou, Jonia Caon, Luiza Gullino e Maíra Barillo. Aos meus queridos amigos do LISV: Juliana Barbosa, Luana Calazans (minha eterna Luluzinha), Josi Rossini e Rodrigo Theófilo. Aos meus amigos do JBRJ: Bruno Carvalho, Carlos Ferreira, Carolina Abramovicz, Carolina Brito, Cilene Jordão, Claudio Fraga, Diana Caldas, Elisabeth Córdula, Elsie Guimarães, Herison Medeiros, João Marcelo Braga, Josimar Külkamp, Marcus Nadruz, Marlon Almeida, Ronaldo Marquete, Suzy Wängler, Talita Antunes, Talvanis Lorenzetti, Thales Ornellas e Zé Fernando. Aos meus amores da Sala 93, Andressa Novaes, Beatriz Valente, Catarina de Carvalho, Celeste Heisecke, Fernanda Fraga, Jaime Duque, Marcus Felippe da Silva, Mary Luz e Renato Xavier Prudêncio, muito obrigado pelo apoio incondicional! Obrigado aos meus amados de Washington, DC: Bort Edwards, Cassandra Degaglia, Dana McCoskey, Dani Fraser, Ingrid Lin, Laura Soul, Manuela Dal Forno, Maria João Martins, Michael Joseph, Michael Silva, Mirian Tsuchiya, Morgan Gostel, Sarah Kuppert, Sílvia Pineda-Muñoz e Vincent Verweij. Aos meus amigos de BA: Aline Stadnik, Fernanda Hurbath, James Lucas Lima, Maria Alves, Nádia Roque, Rodrigo Borges e Tiago Vieira. Aos meus amigos de BH: Adriano Valentin, Alexandra Fagundes, Andrea Almeida, Camila Magalhães, Carol Marques, Igor Ballego, Mariana Duarte, Stéphani Bonifácio, Vagner Bellaver e Victor Leite. Obrigado aos amigos de SP: Aline Possamai Della, Andressa Cabral, Barbara Puglia, Bianca Canestraro, Daniela Almeida, Eduardo Lozano, Emanuelle Santos, Fernanda Sartori Petrongari, Gisele Alves, Jéssica Nayara, Luana Sauthier, Marcelo Kubo, Matheus Colli, Rebeca Laino Gama, Simone Silva e Suzana Ehlin Martins. Obrigado ao Mathias Engels por todas as belíssimas imagens, coletas a apoio. Obrigado ao Timothy Evans e toda sua família, especialmente o pequeno Ben, por me receberem tão calorosamente para passar o Thanksgiving com vocês.

Um agradecimento especial para a minha terapeuta, Ivanilde Sampaio, que me ajudou a manter minha sanidade, e a recobrar a minha sanidade mental, mesmo com minha convivência diária com o ambiente tóxico que é a academia. Você me relembrou que eu sou mais do que apenas meu trabalho e me ajudou a recobrar meu amor próprio. Sou imensamente grato e lhe devo a minha vida! 
Meus mais sinceros agradecimentos à minha enorme e maravilhosa família, de sangue e estendida, por sempre estarem do meu lado e me apoiarem. Em especial, agradeço a: minha mãe, Ester, que nunca deixou de acreditar em mim e me apoiar; meu pai, Mauro, por sempre estar presente na minha vida; aos meus irmãos, Conrado e Donato, por serem meus cúmplices na vida e me lembrando que sempre posso contar com eles; minhas primas e comadres, Carolina e Thais, por estarem sempre presentes na minha vida; minha afilhada, Roberta, por ser a luz da minha vida; aos meus avôs e avós, Eunice, Manoel (in memoriam), Otacílio (in memoriam), Marie e Jorge (in memoriam), por terem me servido de inspiração sobre a importância do trabalho árduo e honesto; e Anna Beatriz Mozer, por ser não só minha cunhada, mas minha amiga. Além disso, agradeço a todos os outros membros de minha família, que devido ao seu enorme tamanho, torna impossível citar um por um e agradecê-los individualmente e da maneira que cada um merece. Amo todos vocês!

Finalmente, agradeço a Rafael Felipe de Almeida, meu amigo, marido, colega de trabalho e orientador honorário. Sou muito feliz contigo e nossos quatro filhotes felinos, Salem, Gesonel, Lupita e Paçoca (mal posso esperar pelos filhotes humanos). Sem você essa tese nunca teria sido concluída! Você esteve ao meu lado em todos os momentos, e fico muito grato de termos escolhido um ao outro para dividirmos toda nossa vida. Você e nosso amor serviram de combustível para me manter funcionando durante os altos e baixos desses últimos cinco anos, e espero ter uma vida longa e feliz ao seu lado, para poder te retribuir por todo o amor, atenção, ajuda e suporte. Separados sempre havia a sensação de não-pertencimento, mas juntos nosso amor, algo que não é nem "eu", nem "você". Juntos nos tornamos alguém maior do que nós dois, passamos a ser mais do que nossos interesses individuais. Por isso, muito obrigado por ser a fúria da minha paciência, por ser a Ruby da minha Sapphire, assim podermos ser o diálogo entre duas metades, e juntos nos Garnet. Te amo!!! 


\section{ÍNDICE}

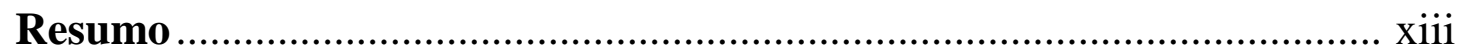

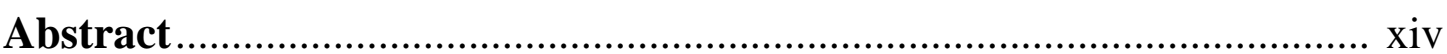

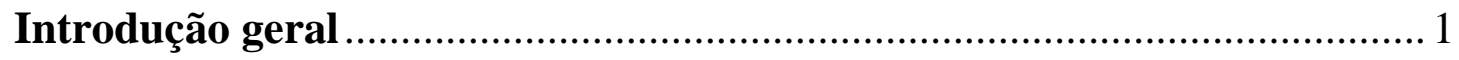

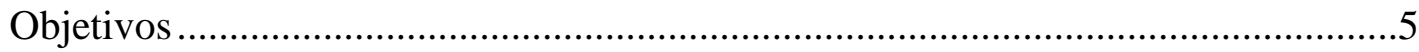

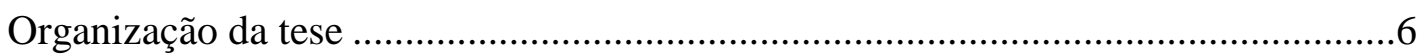

Referências bibliográficas................................................................................

Section 1. Commelinales Mirb. ex Bercht. \& J.Presl..................................... 10

Chapter 1.1. An updated classification of Commelinales (Monocotyledonae) based on molecular and morphological data...................................................................10

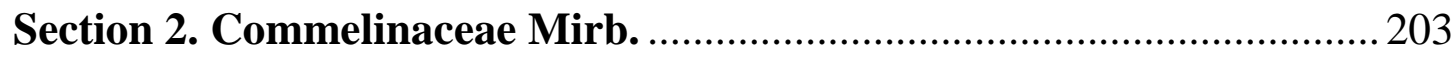

Chapter 2.1. Wandering from Down Under: Late Cretaceous origin of Commelinaceae (Commelinales) in Australian rainforests and the tropical conquering by five major dispersal events ........................................................203

Chapter 2.2. Taxonomic revision of Neotropical Murdannia Royle

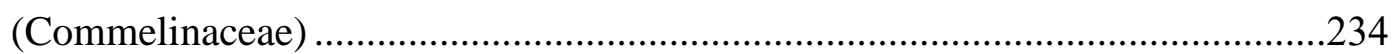

Chapter 2.3. Taxonomic revision of Aneilema sect. Rhopalephora (Hassk.) M.Pell. \& Nandikar, and comments on Aneilema R.Br. s.lat. (Commelinaceae).............277

Chapter 2.4. Synopsis of Commelina L. (Commelinaceae) in the state of Rio de Janeiro, reveals a new white-flowered species endemic to Brazil.........................338

Chapter 2.5. Rediscovery, identity and typification of Dichorisandra picta and comments on the short-stemmed Dichorisandra species 362

Chapter 2.6. Siderasis albofasciata sp. nov. (Commelinaceae), endemic to the state of Espírito Santo, Brazil, and the typification of S. fuscata.

Chapter 2.7. Recircumscription and taxonomic revision of Siderasis, with comments on the systematics of subtribe Dichorisandrinae (Commelinaceae)

Chapter 2.8. Recircumscription and synopsis of Thyrsanthemum Pichon and Weldenia Schult.f. (Commelinaceae), two narrow endemic genera from Mesoamerica

Section 3. Haemodoraceae R.Br. 481

Chapter 3.1. Revisiting the taxonomy of the Neotropical Haemodoraceae (Commelinales) 
Section 4. Pontederiaceae Kunth

Chapter 4.1. Two new synonyms for Heteranthera (Pontederiaceae, Commelinales) .524

Chapter 4.2. Two peculiar new species of Heteranthera Ruiz \& Pavón (Pontederiaceae) from Brazil, with notes on inflorescence architecture in the family .540

Chapter 4.3. Total evidence phylogeny of Pontederiaceae (Commelinales) sheds light on the necessity of its recircumscription and synopsis of Pontederia L. ....564

Conclusões gerais 624

Anexo- The identity and application of Coletia madida and notes on the typification of Mayacaceae 628 


\section{RESUMO}

Commelinales é uma pequena e bem sustentada ordem de monocotiledôneas, atualmente posicionada no clado das Commelinídeas, juntamente com Zingiberales (seu grupo-irmão), Poales, Arecales e Dasypogonales. A ordem é composta por Commelinaceae, Haemodoraceae, Hanguanaceae, Philydraceae e Pontederiaceae, e compreende 60 gêneros e ca. 1080 espécies. Apesar do seu relativamente pequeno número de gêneros e espécies, Commelinales é geografica-, ecológica- e morfologicamente bastante diversa. A ordem apresenta uma distribuição Pantropical, mas alcança regiões temperadas em algumas partes do mundo. A relação entre as cinco famílias de Commelinales é atualmente bem compreendida e fortemente sustentada. Entretanto, Commelinales é a ordem menos estudada dentre as monocotiledôneas do ponto de vista evolutivo e taxonômico. Em sua circunscrição atual, a ordem é exclusivamente circunscrita com base em caracteres moleculares, carecendo de qualquer tipo de suporte morfológico. Assim, no presente trabalho foi realizado um estudo abrangente da morfologia da ordem e suas famílias, visando: (1) apresentar uma nova hipótese filogenética para Commelinales com base em dados combinados; (2) circunscrever morfologicamente Commelinales; e (3) revisitar sua classificação. Como resultado desse estudo são apresentados trabalhos de cunho taxonômico e sistemático para as três famílias ocorrentes no Neotrópico, que também representam as três maiores famílias da ordem (i.e., Commelinaceae, Haemodoraceae e Pontederiaceae), assim como é proposta uma nova classificação para Commelinales com base na combinação de dados moleculares com uma grande matriz morfológica.

Palavras-chave. Commelinídeas, filogenia, monocotiledôneas, morfologia, taxonomia 


\section{ABSTRACT}

Commelinales is a small and well-supported monocot order, currently placed in the commelinid monocots, together with Zingiberales (its sister-group), Poales, Arecales, and Dasypogonales. The order is composed of Commelinaceae, Haemodoraceae, Hanguanaceae, Philydraceae, and Pontederiaceae, and comprises 60 genera and ca. 1,080 species. Despite its relatively small number of genera and species, Commelinales is geographically, ecologically and morphologically very diverse. The order presents a Pantropical distribution, but also reaching temperate regions in some parts of the globe. The relationship between its five families is currently wellunderstood and statistically well-supported. Nonetheless, Commelinales is the least studied monocot order from evolutionary and taxonomic points of view. In its current circumscription, it is solely circumscribed by molecular data, lacking any kind of morphological support. Thus, in the present thesis a comprehensive study of the morphology of the order and its families was carried out, with the aim: (1) presenting a new phylogenetic hypothesis for Commelinales, based on the combination of molecular and morphological data; (2) morphologically circumscribe Commelinales; and (3) revisit its classification. As a result, I provide taxonomic and systematic studies for the three families recorded for the Neotropics, which are also the largest in the order (i.e., Commelinaceae, Haemodoraceae, and Pontederiaceae), together with a new classification for Commelinales, based on the combination of molecular data and an extensive morphological matrix.

Keywords. Commelinid, monocots, morphology, phylogeny, taxonomy 


\section{INTRODUÇÃO GERAL}

Commelinales é uma pequena e bem sustentada ordem de monocotiledôneas, atualmente posicionada no clado das Commelinídeas, juntamente com Zingiberales (seu grupo-irmão), Poales, Arecales e Dasypogonales (Hertweck et al. 2015; Givnish et al. 2018). A ordem é composta pelas famílias Commelinaceae, Haemodoraceae, Hanguanaceae, Philydraceae e Pontederiaceae (Givnish et al. 1999, 2006, 2018; Chase et al. 2000, 2006; Savolainen et al. 2000; Soltis et al. 2000; Graham et al. 2002, 2006; Davis et al. 2004; Saarela et al. 2008; Hertweck et al. 2015; APG IV 2016), compreendendo 60 gêneros e ca. 1080 espécies (Hamann 1966, 1998; Simpson 1990, 1998b; Faden \& Hunt 1991; Bayer et al. 1998; Faden 1998; Leong-Škorničková \& Boyce 2015; Pellegrini 2017a, 2017b; Pellegrini et al. 2018; presente estudo). Apesar do seu relativamente pequeno número de gêneros e espécies, comparado a outras ordens de monocotiledôneas, Commelinales é geografica, ecológica e morfologicamente bastante diversa (Saarela et al. 2008). A ordem apresenta uma distribuição Pantropical, alcançando regiões temperadas em algumas partes do mundo, especialmente devido a ampla distribuição de suas três maiores famílias, Commelinaceae, Haemodoraceae e Pontederiaceae (Stevens 2001-em diante). Em relação às famílias, a Australásia representa o centro de diversidade de Hanguanaceae, Haemodoraceae e Philydraceae (Hamann 1966, 1998; Simpson 1990, 1998b; Bayer et al. 1998). Por outro lado, Pontederiaceae tem os Neotrópicos como centro de diversidade, especialmente o Brasil (Pellegrini \& Horn 2017; Pellegrini et al. 2018). Por último, Commelinaceae apresenta dois centros de diversidade Neotropicais (i.e., México e Brasil; Hunt 1983; Aona 2008; Pellegrini 2017b) e dois Paleotropicais (i.e., África e Ásia; Faden 1991, 2012; Nandikar \& Gurav 2015; Pellegrini et al. 2016).

Commelinales é a ordem menos estudada dentre as monocotiledôneas do ponto de vista evolutivo e taxonômico, tendo sofrido as mudanças mais marcantes em sua composição entre os diferentes sistemas de classificação (Givnish et al. 1999; Chase 2004; Wilkin et al. 2005; Saarela et al. 2008). A única família consistentemente associada à Commelinales foi a sua família-tipo, Commelinaceae, juntamente com Mayacaceae. A maioria das famílias, tradicionalmente posicionadas em Commelinales (i.e., Eriocaulaceae, Mayacaceae, Rapateaceae, Restionaceae e Xyridaceae), está atualmente posicionada em Poales sensu APG (Givnish et al. 1999; Chase 2004; APG 
IV 2016), enquanto Dasypogonaceae é posicionada em Arecales (Hertweck et al. 2015; APG IV 2016) ou aceita em sua própria ordem, Dasypogonales (Givnish et al. 1999, 2018). Por outro lado, as famílias atualmente incluídas em Commelinales foram raramente consideradas como proximamente relacionadas à Commelinaceae (Givnish et al. 1999; Chase 2004). Haemodoraceae, Philydraceae e Pontederiaceae foram geralmente consideradas como, mais ou menos, proximamente relacionadas, apesar de terem sido posicionadas em diferentes ordens ao longo dos vários sistemas de classificação propostos (Simpson 1990; Chase 2004). Além disso, Hanguanaceae foi historicamente considerada uma família de posicionamento incerto dentre as monocotiledôneas (Rudall et al. 1999). Entretanto, a relação entre as cinco famílias de Commelinales é atualmente bem entendida e fortemente sustentada, baseada em vários marcadores nucleares e plastidiais (Chase et al. 2006; Graham et al. 2006; Saarela et al. 2008; Hertweck et al. 2015) e, mais recentemente, com base no genoma completo de cloroplasto (Givnish et al. 2018).

Em sua circunscrição atual, Commelinales era exclusivamente circunscrita com base em caracteres moleculares, carecendo de qualquer tipo de suporte morfológico (Chase 2004; Judd et al. 2008; Saarela et al. 2008; Takhtajan 2009; Soltis et al. 2018), até o presente trabalho. Apesar de alguns estudos sistemáticos terem apontado sinapomorfias morfológicas para famílias individuais em Commelinales (Haemodoraceae: Simpson 1983, 1989, 1990, 1998a; Philydraceae: Simpson 1985, 1990; Pontederiaceae: Simpson 1987, 1990, Simpson \& Burton 2006, Pellegrini et al. 2018), poucos estudos apontaram sinapomorfias para agrupamentos suprafamiliares (i.e., Givnish et al. 1999; Prychid \& Rudall 1999; Saarela et al. 2008; Pellegrini et al. 2018). Ainda, apenas um único estudo anterior a esse apontou uma sinapomorfia morfológica para a ordem (Givnish et al. 1999). Os dois estudos mais abrangentes e importantes sobre a filogenia da ordem são Jesson \& Barrett (2003) e Saarela et al. (2008). Jesson \& Barrett (2003) apresentam uma super-árvore para as monocotiledôneas, com ênfase na ordem Commelinales (Fig 1). A árvore apresentada pelos autores foi construída com base em hipóteses filogenéticas disponíveis na época, tanto para as monocotiledôneas quanto para a ordem 


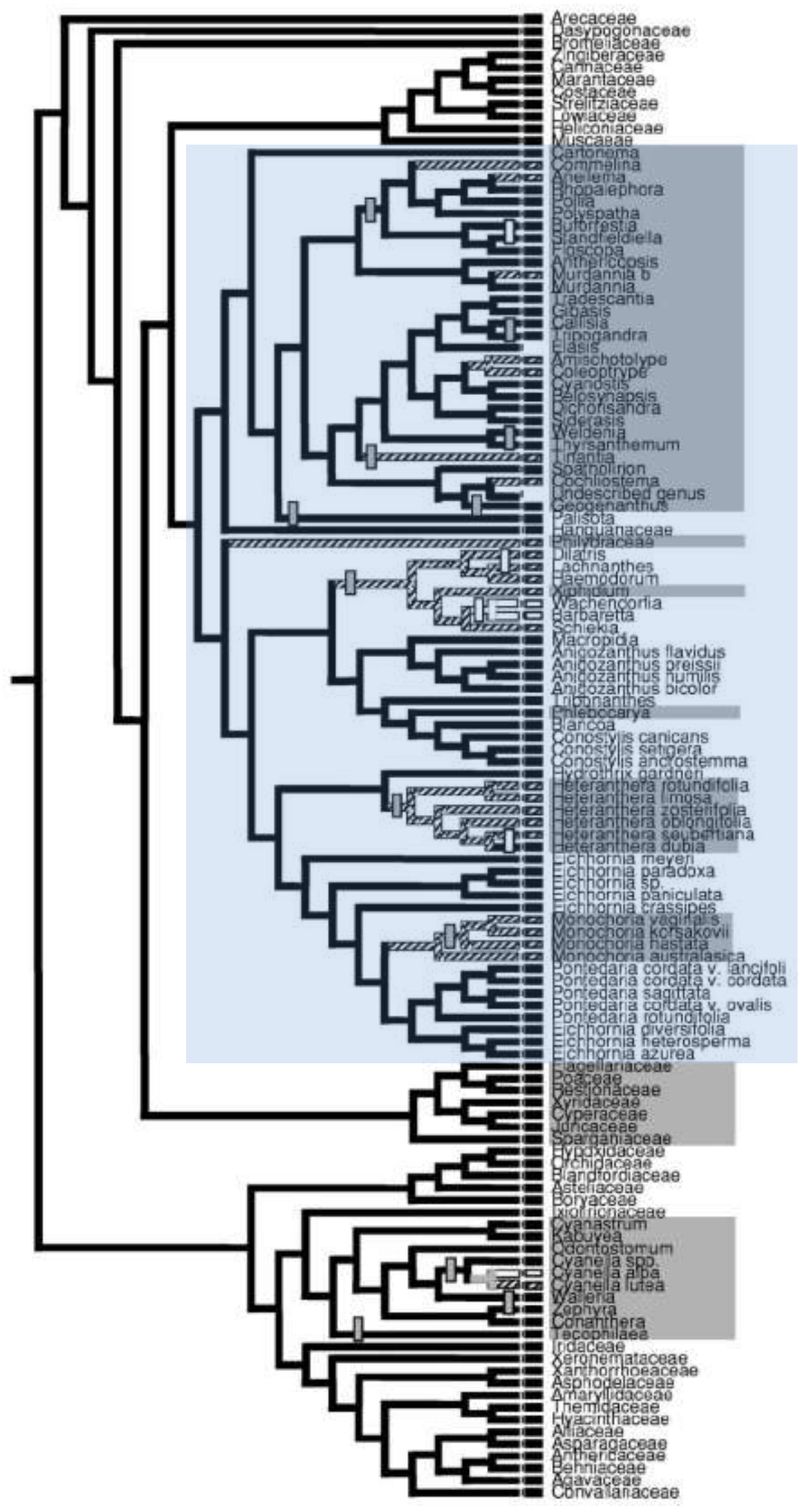

Figura 1. Super-árvore das monocotiledôneas detalhando as relações dentro de Commelinales, mostrando a reconstrução mais parcimoniosa para a perda de nectários, surgimento de heteranteria, e o surgimento de enantiostilia. Commelinales destacada no retângulo azul. Modificado de Jesson \& Barrett (2003). 
Commelinales. Devido à metodologia utilizada, a árvore apresentada não possui valores de suporte estatístico para seus agrupamentos. Mesmo assim, é a hipótese filogenética com a amostragem mais ampla até o momento. Já o trabalho de Saarela et al. (2008) se propôs em apresentar uma filogenia para Commelinales, resolvendo, com grande suporte estatístico, o posicionamento incerto de Philydraceae (Fig 2). Assim, a ordem Commelinales apresenta duas hipóteses filogenéticas baseadas em metodologias distintas. A primeira foi baseada em uma ampla amostragem de táxons dentro da ordem, mas não apresentou suporte estatístico, devido à natureza do método utilizado. Enquanto a segunda apresentou uma topologia com base em vários marcadores moleculares, robustamente sustentada, mas com poucos terminais por família, produzindo assim apenas uma hipótese de backbone ou relações em nível de família, para a ordem. 


\section{Objetivos}

Assim, a presente tese teve como objetivos principais: (1) apresentar uma nova hipótese filogenética para Commelinales com base na combinação de dados moleculares e uma extensa matriz morfológica; (2) circunscrever morfologicamente Commelinales; e (3) revisitar a classificação de Commelinales com base nos resultados obtidos, propondo uma nova classificação, composta por grupos monofiléticos e com coerência morfológica. Para isso, foi necessário um amplo e aprofundado estudo de todas as famílias e suas principais linhagens. Os objetivos específicos dessa tese, estão relacionados com os problemas taxonômicos dentro das famílias de Commelinales e foram: (1) refinar a filogenia e apresentar a primeira hipótese biogeográfica para Commelinaceae; (2) revisar alguns gêneros com posicionamento chave dentro de Commelinaceae (i.e., Gibasoides D.R.Hunt., Thyrsanthemum Pichon, Rhopalephora

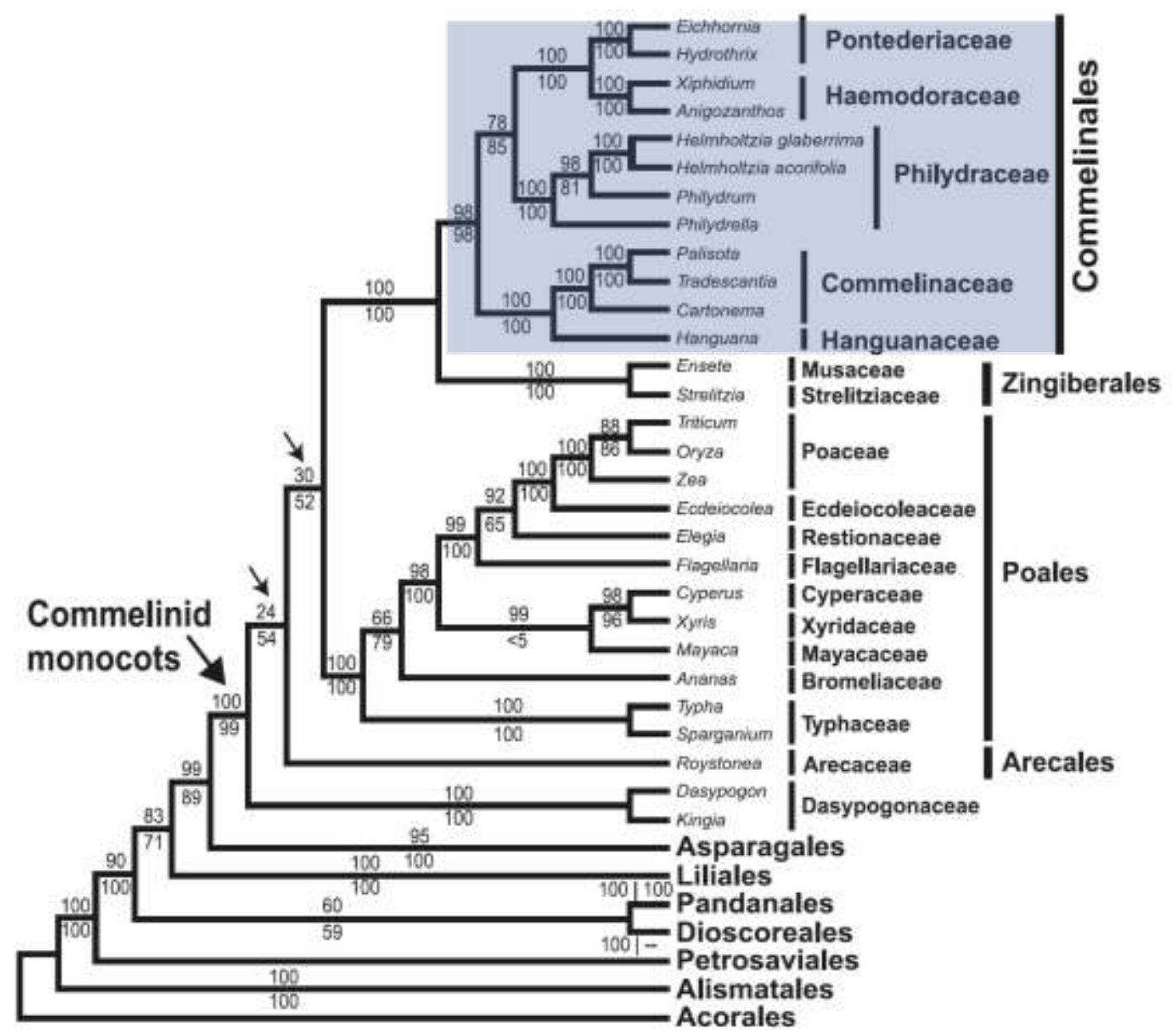

Figura 2. Filogenia para as monocotiledôneas com base em 17 loci plastidiais e regiões não-codificantes associadas. Valores acima dos ramos apresentam o valor de suporte (bootstrap) da análise de Parcimônia, enquanto abaixo representam o valor de suporte (bootstrap) da análise de Máxima Verossimilhança. Ramos com setas colapsaram no consenso estrito. Commelinales destacada no retângulo azul. Modificado de Saarela et al. (2008). 
Hassk., Siderasis Raf. e Weldenia Schult.f.) e grupos de espécies dentro de gêneros (i.e., Commelina L., Dichorisandra J.C.Mikan, e Murdannia Royle); (3) revisitar a taxonomia das Haemodoraceae Neotropicais; (4) nova circunscrição para os gêneros de Pontederiaceae, com base em dados morfológicos e moleculares; e (5) descrever os novos táxons encontradas ao longo da elaboração do tratamento taxonômico para Commelinales.

\section{Organização da tese}

A tese está organizada em quatro seções, cada uma subdividida em um ou mais capítulos:

- Seção 1. Commelinales- Essa seção encontra-se dividida em apenas um capítulo, intitulado "An updated classification of Commelinales (Monocotyledonae) based on molecular and morphological data". Este capítulo está diretamente relacionado aos objetivos principais dessa tese, representando a compilação de todos os estudos realizados durante o desenvolvimento dessa tese.

- Seção 2. Commelinaceae- Essa seção encontra-se dividida em oito capítulos, que abordam problemas taxonômicos dentro das duas principais linhagens de Commelinaceae (i.e., tribos Commelineae e Tradescantieae), assim como apresentam uma nova hipótese filogenética para a família, datada e calibrada, juntamente com sua primeira hipótese biogeográfica. Os gêneros Rhopalephora e Siderasis foram revisados, assim como as espécies Neotropicais de Murdannia, enquanto para os gêneros Gibasoides, Thyrsanthemum e Weldenia são apresentadas sinopses. Para o gênero Commelina é apresentada uma sinopse para o estado do Rio de Janeiro, com a descrição de uma nova espécie. Enquanto para Dichorisandra, o grupo de espécies acaules foi investigado, com ênfase na sua morfologia e variação floral, dentro do contexto da revisão de seu grupo-irmão (i.e., Siderasis).

- Seção 3. Haemodoraceae- Essa seção encontra-se dividida em apenas um capítulo, intitulado "Revisiting the taxonomy of the Neotropical Haemodoraceae (Commelinales)". Este capítulo revisita a taxonomia das Haemodoraceae Neotropicais, solucionando problemas que ficaram pendentes desde o tratamento da família para a obra Flora Neotropica. 
- Seção 4. Pontederiaceae- Essa seção encontra-se dividida em três capítulos, abordando os limites genéricos e arquitetura de inflorescência em Pontederiaceae. No primeiro capítulo, o gênero Heteranthera Ruiz \& Pav. é expandido para incluir Hydrothrix Hook.f. e Scholleropsis H.Perrier. No segundo capítulo, a arquitetura de inflorescência de Pontederiaceae é revisitada, dentro do contexto da descrição de duas espécies peculiares para o Brasil. Por último, o terceiro capítulo conclui e re-delimitação genérica de Pontederiaceae, com base em análise combinada de dados morfológicos e moleculares. Nesse contexto, Pontederia L. é expandida para incluir Eichhornia Kunth e Monochoria C.Presl.

\section{Referências bibliográficas}

Aona LYS (2008) Revisão taxonômica e análise cladística do gênero Dichorisandra J.C.Mikan (Commelinaceae). Ph.D. thesis. Instituto de Biologia, Universidade Estadual de Campinas, Campinas, São Paulo, Brazil.

APG IV - The Angiosperm Phylogeny Group (2016) An update of the Angiosperm Phylogeny Group classification for the orders and families of flowering plants: APG IV. Bot. J. Linn. Soc. 181: 1-20.

Backer CA (1951) Hanguana (Flagellariaceae). In: Flora Malesiana 4: 248-250.

Bayer C, Appel O, Rudall PJ (1998) Hanguanaceae. In: Kubitzki K (ed.) The families and genera of vascular plants, vol. 4. Springer Verlag, Berlin, Germany, Pp. 223225.

Chase MW, Fay MF, Devey DS, Maurin O, Rønsted N, Davies TJ, Pillon Y, Petersen G, Seberg O, Tamura MN, Asmussen CB, Hilu K, Borsch T, Davis JI, Stevenson DW, Pires JC, Givnish TJ, Sytsma KJ, McPherson MA, Graham SW, Rai HS (2006) Multigene analyses of monocot relationships: a summary. In: Columbus JT et al. (eds.) Monocots: comparative biology and evolution (excluding Poales). Rancho Santa Ana Botanic Garden, Claremont, California, USA, Pp. 63-75.

Chase MW (2004) Monocot relationships: an overview. American Journal of Botany 91: $1645-1655$.

Chase MW, Soltis DE, Soltis PS, Rudall PJ, Fay MF, Hahn WH, Sullivan S, Joseph J, Molvray M, Kores PJ, Givnish TJ, Sytsma KJ, Pires JC (2000) Higher-level systematics of the monocotyledons: an assessment of current knowledge and a new classification. In: Wilson KL \& Morrison DA (eds.) Monocots: systematics and evolution. CSIRO, Collingwood, Victoria, Australia, Pp. 3-16.

Davis JI, Stevenson DW, Peterson G, Seberg O, Campbell LM, Freudenstein JV, Goldman DH, Hardy CR, Michelangeli FA, Simmons MP, Specht CD, VergaraSilva F, Gandolfo MA (2004) A phylogeny of the monocots, as inferred from $r b c L$ 
and atpA sequence variation. Syst. Bot. 29: 467-510. doi:10.1600/0363644041744365.

Faden RB (1991) The morphology and taxonomy of Aneilema R.Brown (Commelinaceae). Smithsonian Contributions to Botany 76: 1-181.

Faden RB (1998) Commelinaceae. In: Kubitzki K (ed.) The families and genera of vascular plants, vol. 4. Springer Verlag, Berlin, Germany, 109-128.

Faden RB (2012) Commelinaceae. In: Beentje HJ (ed.) Flora of East Tropical Africa. Royal Botanic Gardens, Kew, London. 1-244.

Givnish TJ, Evans TM, Pires JC, Sytsma KJ (1999) Polyphyly and convergent morphological evolution in Commelinales and Commelinidae: evidence from $r b c L$ sequence data. Molecular phylogenetics and Evolution. 12: 360-385.

Givnish TJ, Pires JC, Graham SW, McPherson MA, Prince LM, Patterson TB, Rai HS, Roalson EH, Evans TM, Hahn WJ, Millam KC, Meerow AW, Molvray M, Kores PJ, O’Brien HE, Hall JC, Kress WJ, Sytsma KJ (2006) Phylogenetic relationships of monocots based on the highly informative plastid gene ndhF: evidence for widespread concerted convergence. In: Edited by Columbus JT et al. (eds.) Monocots: comparative biology and evolution (excluding Poales). Rancho Santa Ana Botanic Garden, Claremont, California, USA, Pp. 28-51.

Givnish TJ, Zuluaga A, Spalink D, Gomez MS, Lam VKY, Saarela JM, Sass C, Iles WJD, Sousa DJL, Leebens-Mack J, Pires JC, Zomlefer WB, Gandolfo MA, Davis JI, Stevenson DW, dePamphilis C, Specht CD, Graham SW, Barrett CF, Ané C (2018) Monocot plastid phylogenomics, timeline, net rates of species diversification, the power of multi-gene analyses, and a functional model for the origin of monocots. American Journal of Botany 105: 1888-1910.

Graham SW, Olmstead RG, Barrett SCH (2002) Rooting phylogenetic trees with distant outgroups: a case study from the commelinoid monocots. Mol. Biol. Evol. 19: 1769-1781. PMID:12270903.

Hamann U (1966) Embryologische, morphologischanatomische und systematische Untersuchungen an Philydraceen. Willdenowia, Beih. 4: 1-178, 8 tab.

Hamann U (1998) Philydraceae. In: Kubitzki K (ed.) The families and genera of vascular plants, vol. 4. Springer Verlag, Berlin, Germany, Pp. 389-394.

Hertweck KL, Kinney MS, Stuart SA, Maurin O, Mathews S, Chase MW, Gandolfo MA, Pires JC (2015) Phylogenetics, divergence times and diversification from three genomic partitions in monocots. Bot. J. Linn. Soc. 178: 375-393.

Hunt DR (1983) The taxonomy of the tribe Tradescantieae (Commelinaceae). Ph.D. thesis. The University of Reading, Reading, UK.

Jesson LK, Barrett SCH (2003) The comparative biology of mirror-image flowers. International Journal of Plant Science 164: S237-S249. 
Leong-Škorničková J, Boyce PC (2015) Hanguana in Singapore demystified: an overview with descriptions of three new species and a new record. Gardens' Bulletin Singapore 67: 1-28.

Nandikar MD, Gurav RV (2015) Revision of the genus Murdannia (Commelinaceae) in India. Phytodiversity 2: 56-112.

Pellegrini MOO (2017a) Two new synonyms for Heteranthera (Pontederiaceae, Commelinales). Nordic Journal of Botany 35: 124-128. https://doi.org/10.1111/njb.01152

Pellegrini MOO (2017b) Morphological phylogeny of Tradescantia L. (Commelinaceae) sheds light on a new infrageneric classification for the genus and novelties on the systematics of subtribe Tradescantiinae. PhytoKeys 89: 11-72. https://doi.org/10.3897/phytokeys.89.20388

Pellegrini MOO, Faden RB (2017) Recircumscription and taxonomic revision of Siderasis, with comments on the systematics of subtribe Dichorisandrinae (Commelinaceae). PhytoKeys 83: 1-41. https://doi.org/10.3897/phytokeys.83.13490

Pellegrini MOO, Horn CN (2017) Two peculiar new species of Heteranthera Ruiz \& Pavón (Pontederiaceae) from Brazil, with notes on inflorescence architecture in the family. PhytoKeys 82: 35-56. https://doi.org/10.3897/phytokeys.82.13752

Pellegrini MOO, Horn CN, Almeida RF (2018) Total evidence phylogeny of Pontederiaceae (Commelinales) sheds light on the necessity of its recircumscription and synopsis of Pontederia L. PhytoKeys 108: 25-83. https://doi. org/10.3897/phytokeys.108.27652

Prychid CJ, Rudall PJ (1999) Calcium oxalate crystals in monocotyledons: a review of their structure and systematics. Ann. Bot. (Lond.) 84: 725-739. doi:10.1006/anbo.1999.0975.

Rudall PJ, Stevenson DW, Linder HP (1999) Structure and systematics of Hanguana, a Monocotyledon of uncertain affinity. Austr. Syst. Bot. 12: 311-330.

Saarela JM, Prentis PJ, Rai HS, Graham SW (2008) Phylogenetic relationships in the monocot order Commelinales, with a focus on Philydraceae. Botany (Canada) 86: 719-731.

Savolainen V, Chase MW, Hoot SB, Morton CM, Soltis DE, Bayer C, Fay MF, de Bruijn AY, Sullivan S, Qiu Y-L (2000) Phylogenetics of flowering plants based on combined analysis of plastid atpB and $r b c L$ gene sequences. Syst. Biol. 49: 306362. doi:10.1080/10635159950173861. PMID:12118410.

Simpson MG (1985) Pollen ultrastructure of the Philydraceae. Grana 24: 23-31.

Simpson MG (1987) Pollen ultrastructure of the Pontederiaceae. Grana 26: 113-126.

Simpson MG (1990) Phylogeny and classification of the Haemodoraceae. Annals of the Missouri Botanical Garden 77(4): 722-784, pl. XXI-XXIII. 
Simpson MG (1998b) Haemodoraceae. In: Kubitzki K (Ed) The families and genera of vascular plants, vol. 4. Springer Verlag. Berlin, Germany, Pp. 212-128.

Simpson MG, Burton DH (2006) Systematic floral anatomy of Pontederiaceae. Aliso 22: 499-519.

Stevens PF (2001-onwards) Angiosperm Phylogeny Website. Version 14, July 2017 [and more or less continuously updated since]. Available from: $<$ http://www.mobot.org/MOBOT/research/APweb/>. [accessed: 6.1.2019]

Takhtajan A (2009) Flowering Plants. 2 ed. Springer Science+Business Media B.V., Berlin, Germany.

Wilkin P, Schols P, Chase MW, Chayamarit K, Furness CA, Huysmans S, Rakotonasolo F, Smets E, Thapyai C (2005) A plastid gene phylogeny of the yam genus, Dioscorea: Roots, fruits and Madagascar. Syst. Bot. 30: 736-749. 


\section{CONSIDERAÇÕES FINAIS}

O presente trabalho apresentou a mais bem sustentada e amostrada hipótese filogenética para Commelinales. Essa foi a primeira vez que uma hipótese baseada exclusivamente em dados morfológicos foi proposta, além de ser a primeira vez que uma hipótese com base na combinação de dados morfológicos e moleculares também foi proposta. Entretanto, a presente hipótese filogenética não é de maneira alguma final e maiores estudos e esforços filogenômicos e morfológicos serão necessários para esclarecer pontos ainda não completamente solucionados. Os dados moleculares disponíveis no GenBank para as cinco famílias de Commelinales, mais as novas sequências produzidas ainda se mostram insuficientes devido à grande quantidade de dados faltantes nos vários marcadores que foram investigados para apenas uma ou poucas famílias da ordem. Assim, ainda é necessário homogeneizar a amostragem de marcadores entre as cinco famílias para uma hipótese molecular ainda mias robusta e bem sustentada, principalmente em relação ao backbone de Commelinales, possa ser apresentada. Em relação aos dados morfológicos, a presente matriz contém 570 caracteres, que abrangeram dados macro- e micromorfológicos, citogenéticos e fitoquímicos. Apesar da enorme congruência entre o presente conjunto de dados morfológicos e os dados moleculares, incongruências ou baixa resolução interna foram observadas dentro de grupos específicos, tais como: Callisia Loefl., Gibasis Raf. e Tripogandra Raf. (Commelinaceae), Hanguanaceae, Anigozanthos Labill., Conostylis R.Br., Haemodorum Sm. e Tribonanthes Endl. (Haemodoraceae), e Philydraceae. Assim, a inclusão de novos caracteres é essencial para tentar solucionar esses problemas. Estudos anatômicos (tanto de órgãos vegetativos quando de reprodutivos), palinológicos, citogenéticos, fitoquímicos e de morfologia de frutos, sementes e plântulas se mostraram essenciais para aumentar a congruência entre os dados morfológicos e moleculares, além de auxiliar a refinar as hipóteses filogenéticas dentro doss grupos. Caracteres essenciais a serem estudados em Commelinales são: (1) arquitetura vascular caulinar; (2) ontogenia foliar, especialmente em Haemodoraceae, Philydraceae e Pontederiaceae para entender a reversão de folhas unifaciais para bifaciais na última família; (3) anatomia floral, focando na morfologia de células de tanino; (4) ontogenia floral para entendimento do surgimento da enantiostilia, com foco especial em Hanguanaceae; (5) anatomia de 
anteras, focando especialmente na morfologia do tapete; (6) anatomia do gineceu, focando na morfologia da placenta e (7) morfologia e distribuição de cristais de oxalato de cálcio em órgãos vegetativos e reprodutivos. Acredita-se que com esse refinamento será possível propor uma hipótese filogenética ainda mais robusta, que assim poderá ser datada e calibrada. Com base nessa filogenia datada e calibrada será possível investigar a história biogeográfica da ordem, além de permitir o desenvolvimento de um estudo de otimização de caracteres morfológicos e ecológicos chave.

Em reação às famílias de Commelinales, com as contribuições da presente tese, Pontederiaceae passa a se tornar a família mais bem compreendida da ordem. Pontederiaceae apresenta o maior número de hipóteses filogenéticas, com diferentes conjunto de dados mostrando grande congruência entre eles. Além disso, Pontederia L. já foi parcialmente revisado, com tratamentos taxonômicos disponíveis para $P$. subg. Monochoria e $P$. subg. Pontederia, bem como uma sinopse para todo o gênero. Já para Heteranthera Ruiz \& Pav. s.str. há uma revisão taxonômica não-publicada, que está em processo de atualização para abranger a circunscrição atual do gênero e incluir outras novidades taxonômicas. Entretanto, estudos anatômicos, citogenéticos e fitoquímicos ainda são necessários para preencher as lacunas na matriz morfológica para a ordem. Finalmente, a família carece de uma filogenia datada e calibrada e, consequentemente, uma hipótese biogeográfica.

A segunda família mais bem estudada da ordem é Commelinaceae, com enorme número de trabalhos taxonômicos, anatômicos e citológicos publicados. Entretanto, devido ao seu tamanho, boa parte de seus gêneros ainda carece de revisão taxonômica, especialmente os maiores gêneros da família, tais como: Aneilema R.Br., Commelina L., Cyanotis D.Don, Dichorisandra J.C.Mikan, Murdannia Royle e Tradescantia L. emend M.Pell., além de gêneros com posicionamento filogenético crítico (e.g., Cartonema R.Br., Dictyospermum Wight, Floscopa Lour., Palisota Rchb. ex Endl., Pollia Thunb. e Tinantia Scheidw.). Também devido ao seu tamanho, trabalhos focando na anatomia vegetativa e reprodutiva, palinologia, citogenética, fitoquímica e morfologia de plântulas e sementes são necessários para amostrar gêneros e espécies não estudados e preencher as lacunas na matriz morfológica da ordem. Nessa tese foi apresentada a primeira filogenia datada e calibrada para Commelinaceae, juntamente com a primeira hipótese biogeográfica para a família. 
Entretanto, uma amostragem mais completa, de grupos com grande diversidade de espécies e ampla distribuição, é necessária para refinar nossa hipótese biogeográfica. Além disso, também é preciso incluir no conjunto de dados moleculares os gêneros Gibasoides D.R.Hunt, Matudanthus D.R.Hunt e, especialmente, Triceratella Brenan para obtenção de uma hipótese consistente, uma vez que os dados deste último gênero serão decisivo para as análises biogeográficas.

Haemodoraceae recebeu bastante atenção nos últimos 40 anos. Entretanto, boa parte de seus gêneros carecem de revisões taxonômicas, uma vez que os dados disponíveis aparecem apenas em tratamentos florísticos. Por melhores que sejam essas floras, grupos grandes e complicados como Anigozanthos, Conostylis e Haemodorum necessitam de uma revisão taxonômica completa e detalhada. Além disso, gêneros pequenos, tais como: Dilatris P.J.Bergius, Paradilatris (Hopper ex J.C.Manning) Hopper et al., Phlebocarya R.Br., Wachendorfia Burm. e Xiphidium Aubl. necessitam de revisões taxonômicas para que saibamos ao certo quantas espécies os compõem. A família como um todo é bem entendida do ponto de vista filogenético, anatômico, citológico e palinológico. Entretanto, vários caracteres anatômicos incluídos na matriz morfológica apresentada no presente estudo da ordem foram investigados para poucos grupos (e.g., morfologia de estigmas e suas papilas) ou apenas superficialmente investigados (e.g., morfologia de tricomas). Uma nova hipótese filogenética para Haemodoraceae está em fase final de elaboração e este trabalho apresentará uma filogenia datada e calibrada, além de uma primeira hipótese biogeográfica para a família, bem como amostrará todos os gêneros aceitos.

Philydraceae é a menor família da ordem, com quatro gêneros e oito espécies. Durante a elaboração desta tese foi necessário reestabelecer o gênero Orthothylax (Hook.f.) Skottsb., além de reconhecer uma segunda espécie de Philydrum Banks \& Sol. ex Gaertn., bom como uma terceira espécie de Philydrella Caruel. A família apresenta um número relativamente grande de trabalhos investigando diferentes aspectos de sua biologia, como taxonomia, anatomia, palinologia, morfologia de plântulas e sementes, e citogenética. Entretanto, o grupo carece de um tratamento taxonômico moderno, que foi iniciado durante o desenvolvimento dessa tese e que será concluído e publicado posteriormente, além de estudos fitoquímicos e de alguns aspectos anatômicos. Esses caracteres são necessários para resolver as incongruências entre a morfologia e os dados moleculares disponíveis atualmente. Além disso, é 
necessário amostrar molecularmente todas as espécies de Philydraceae, para que se possa propor uma hipótese filogenética robusta e conclusiva.

Hanguanaceae é, ainda, a família menos estudada e compreendida de Commelinales, apesar das inúmeras contribuições para a taxonomia do grupo nos últimos anos. Inúmeras espécies ainda precisam ser descritas, permitindo com isso o avanço dos estudos taxonômicos na família, além de estudos em outras áreas. Estudos fitoquímicos são esparsos em Hanguanaceae e pouco conclusivos devido aos problemas taxonômicos no grupo. Estudos anatômicos, palinológicos, citogenéticos e filogenéticos são pontuais ou inexistentes, geralmente amostrando apenas uma única espécie que muitas vezes não está nem identificada ao nível de espécie. Hanguanaceae é bastante peculiar morfologicamente e necessita de estudo detalhados sobre sua macromorfologia, anatomia vegetativa e reprodutiva, palinologia, citogenética, morfologia de plântulas e sementes, ontogenia floral, e biologia reprodutiva. Esses estudos são essenciais para a taxonomia do grupo em si, mas também para viabilizar o melhor entendimento da ordem Commelinales e de sua relação com Commelinaceae.

Finalmente, a presente tese representou uma contribuição significativa para os estudos em Commelinales, mas está longe de ser final. Os resultados aqui apresentados responderam algumas perguntas sobre o grupo, mas levantaram um número ainda maior de questionamentos. Assim, esta tese foi um pontapé inicial em uma longa série de estudos colaborativos e interdisciplinares em desenvolvimento sobre a ordem Commelinales. 


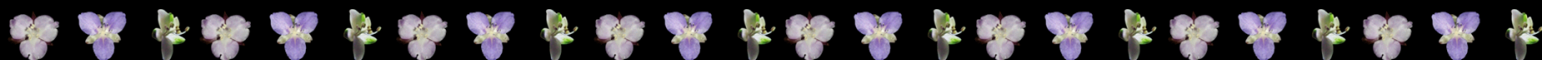

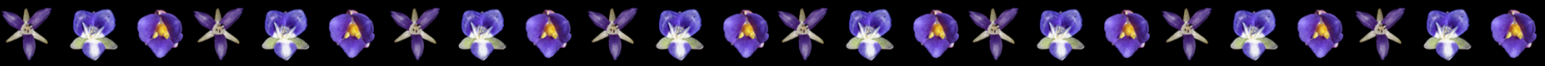
웅

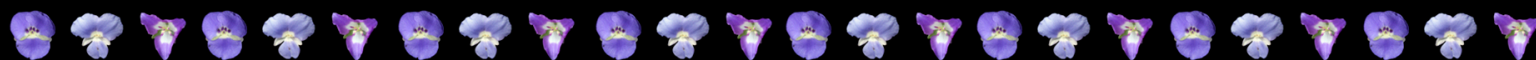
* \%

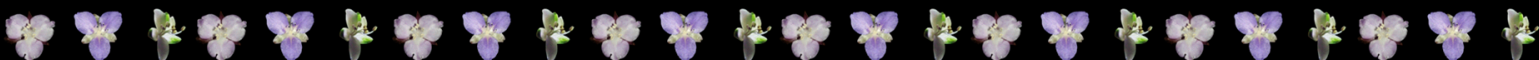

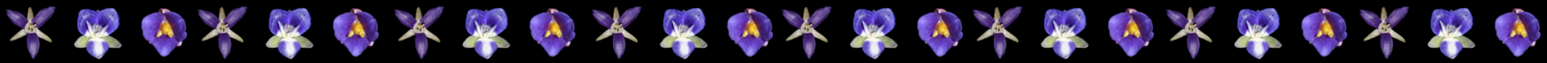

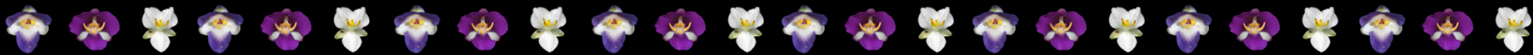

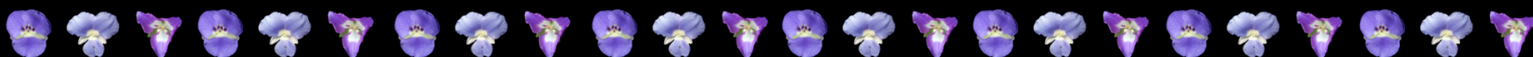

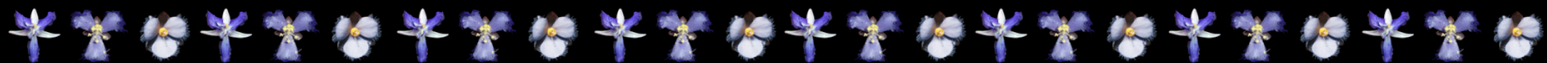

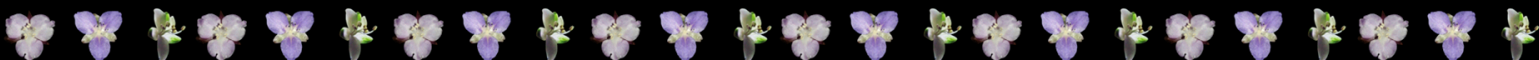

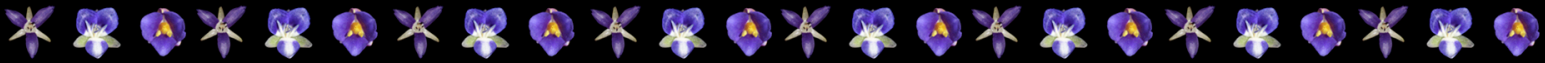

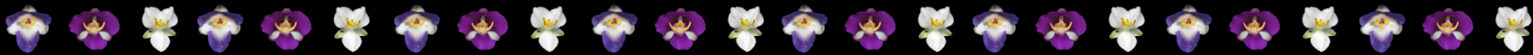

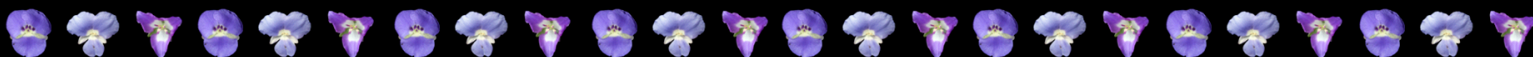

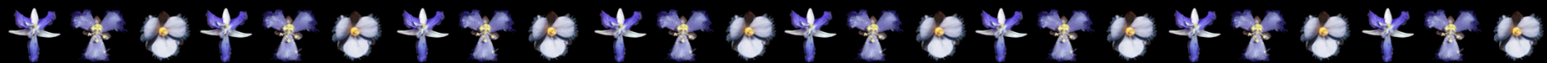

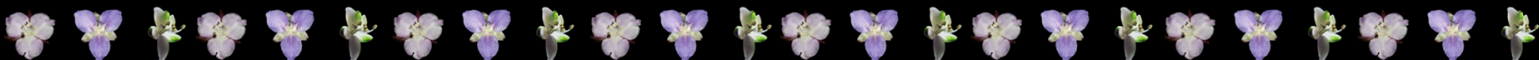

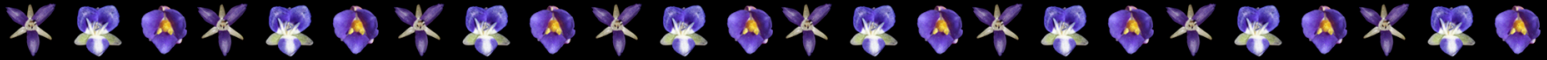
앙 0 \%

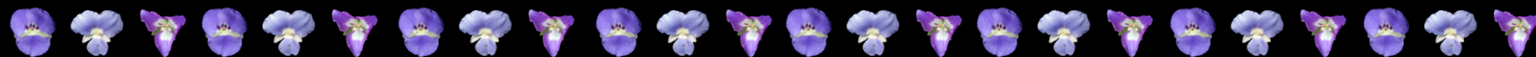

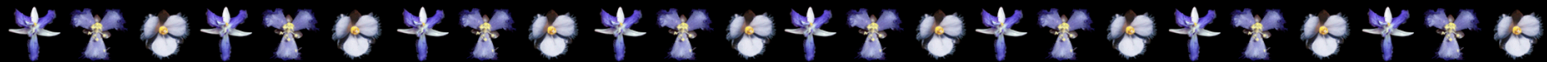

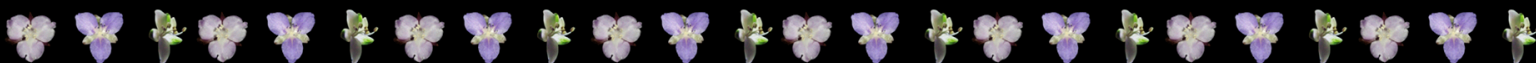

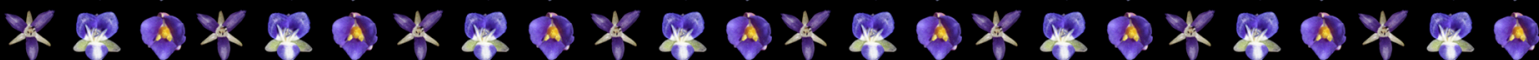

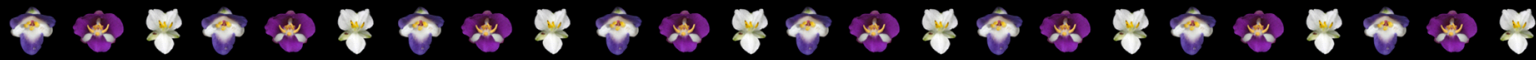

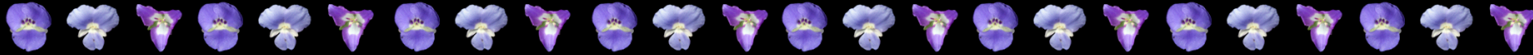

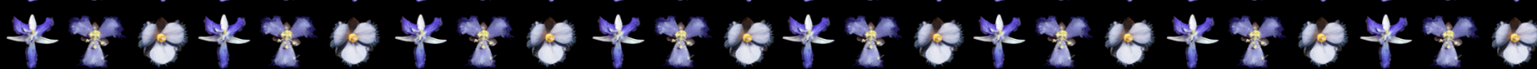

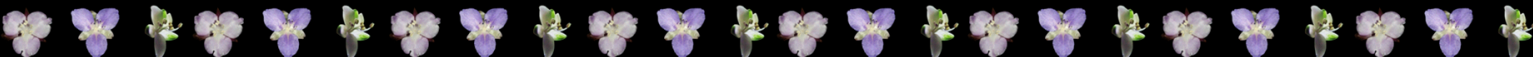

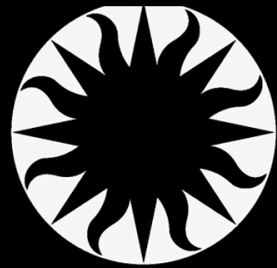

Smithsonian

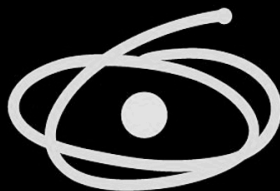

CAPES

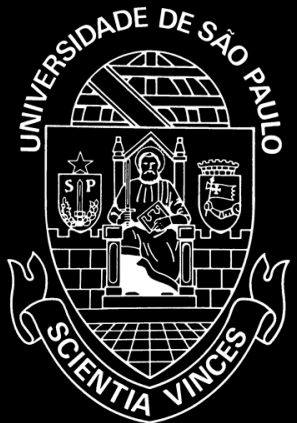

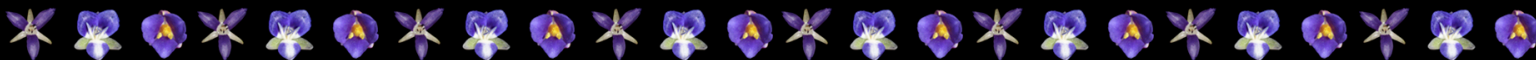
앙

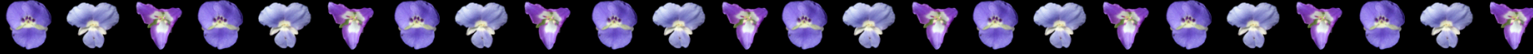

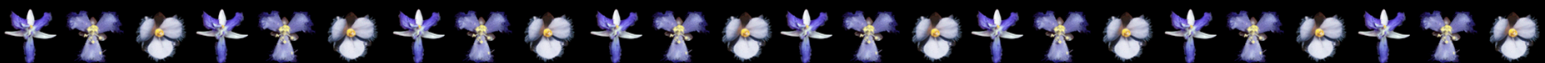

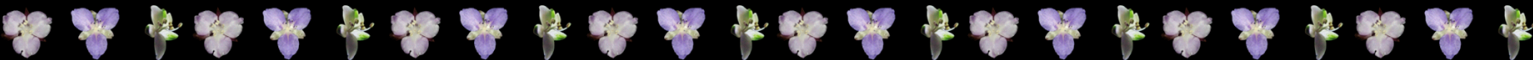

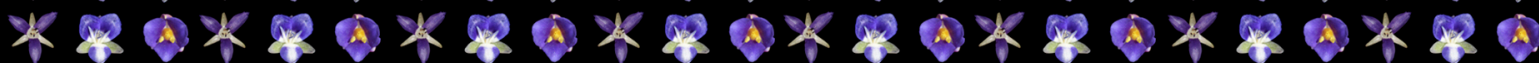

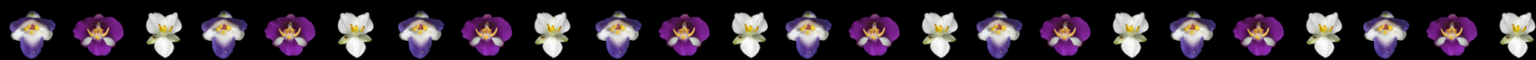

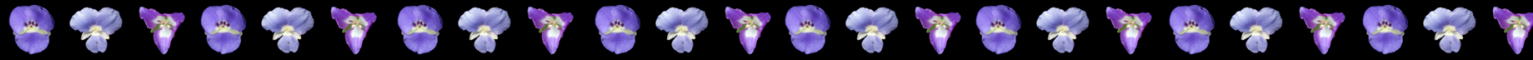

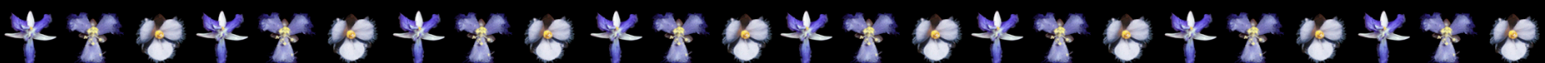

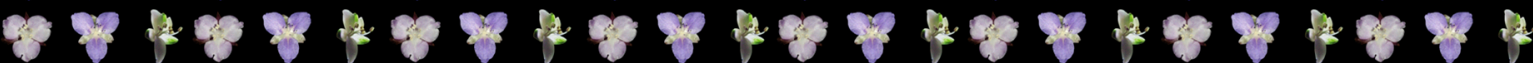

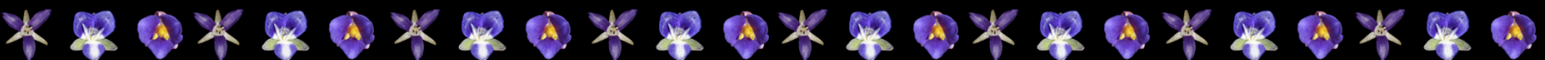
○ * $\%$ \%

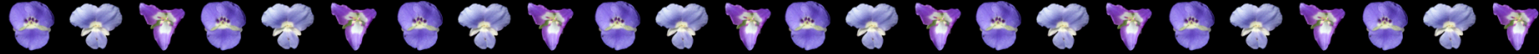

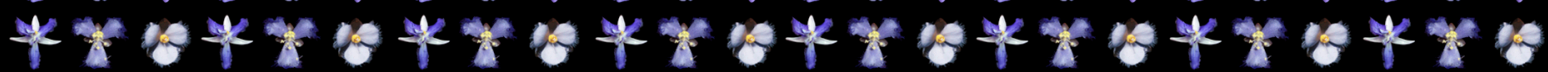

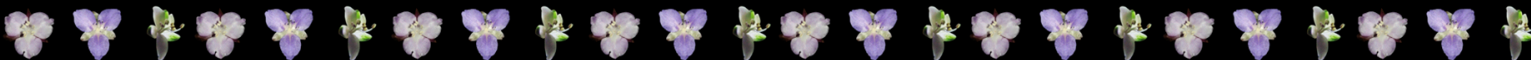

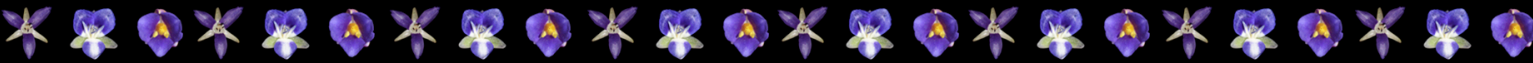

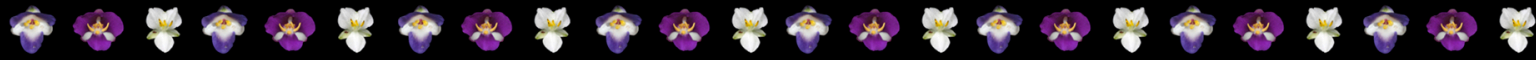

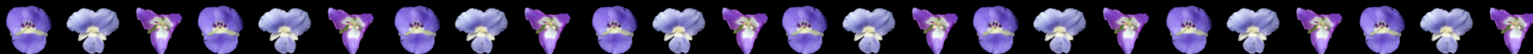

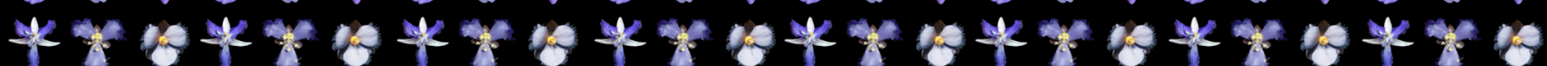

